\title{
Nociceptive pain
}

Clifford J. Woolf

\section{Source}

CliffordJ. Woolf. (2010). What is this thing called pain?. J. Clin. Invest., vol. 120 (11), 37423744. doi:10.1172/jci45178.

Nociceptive pain represents the sensation associated with the detection of potentially tissue-damaging noxious stimuli and is protective. 